\title{
Severe Eosinophilic Colitis Caused By Neuropathic Agents in a Patient with Chronic Fatigue Syndrome and Functional Abdominal Pain: Case Report and Review of the Literature
}

\author{
Konstantinos C. Fragkos, ${ }^{1}$ John Barragry, ${ }^{1}$ Charisma Shahi Fernando, ${ }^{2}$ Marco Novelli, ${ }^{2}$ \\ Joanna Begent, ${ }^{3}$ Natalia Zárate-Lopez ${ }^{1}$ \\ ${ }^{1}$ GI Physiology Unit, Department of Gastroenterology, University College London Hospitals \\ NHS Foundation Trust, 250 Euston Road, London NW1 2PG, United Kingdom \\ ${ }^{2}$ Histopathology Department, University College London Hospitals NHS Foundation Trust, \\ 250 Euston Road, London NW1 2PG, United Kingdom \\ ${ }^{3}$ Adolescent Unit, University College London Hospitals NHS Foundation Trust, 250 Euston \\ Road, London NW1 2PG, United Kingdom
}

Correspondence to:

Natalia Zárate-Lopez

GI Physiology Unit, Department of Gastroenterology

University College London Hospitals NHS Foundation Trust

250 Euston Road, London NW1 2PG, United Kingdom

e-mail: N.Zarate-Lopez@nhs.net

Tel. +44 (0) 20344 79130, Fax: +44 (0) 2034479217

Running head: Neuropathic agents and eosinophilic colitis

Financial support: None.

Conflicts of interest: None.

Patient consent: Yes

Author contributions: KCF, JB and CSF prepared the manuscript and collected clinical data and references; NZL, JB and MN edited and supervised the preparation of the manuscript. 


\section{English Abstract}

Eosinophilic colitis is a rare clinical condition that belongs to the group of eosinophilic gastrointestinal disorders. Its occurrence can be primary or secondary to infection, medications or autoimmune/haematological conditions. We present a case of a young female adult with severe chronic fatigue syndrome, widespread chronic pain, including functional abdominal pain who developed severe eosinophilic colitis following successive treatments with gabapentin and pregabalin. On both occasions, symptoms manifested as abdominal pain, diarrhoea and eosinophilia and improved upon discontinuation of the medications. MRI small bowel demonstrated an ascending colon colitis and endoscopic investigations confirmed florid colitis mainly in the ascending colon with biopsies demonstrating a dense eosinophilic infiltrate with micro-abscesses. Serum eosinophil counts correlated well with the timing of the agents' administration. There was no other organ involvement. Symptoms improved upon discontinuation of the drugs and steroid administration. Eosinophilic colitis is an exceptionally rare entity and its mechanism of action is still unclear. Suspicion of eosinophilic colitis should be raised if a patient presents with abdominal pain, diarrhoea and peripheral eosinophilia following treatment with pregabalin or gabapentin.

\section{Keywords}

gabapentin, pregabalin, eosinophilic colitis, myalgic encephalopathy, functional bowel disorder

\section{German Abstract}

Die eosinophile Kolitis ist eine sehr seltene Krankheit aus der Gruppe der eosinophilen MagenDarm-Erkrankungen. Als Auslöser gelten Primär- und Sekundärinfektionen, Medikamente, sowie autoimmune und hämatologische Erkrankungen. Unser Fall beschreibt eine junge, weibliche Erwachsene mit schwerem chronischem Müdigkeitssyndrom und generalisierten chronischen Schmerzen, darunter funktionellen Unterleibsschmerzen, welche unter Behandlung mit Gabapentin und Pregabalin an einer schweren eosinophilen Kolitis erkrankte. $\mathrm{Zu}$ zwei unterschiedlichen Zeitpunkten wies die Patientin abdominelle Schmerzen, Diarrhoe und ein Eosinophilie auf, mit jeweils Verbesserung der Symptomatik nach Sistieren der Medikamenteneinnahme. In einer Kernspintomographie stellte sich eine Kolitis des aufsteigenden Kolons dar. Endoskopisch konnte eine floride Kolitis, vorwiegend im Kolon ascendens, bestätigt werden, mit Nachweis einer dichten eosinophilen Infiltration mit Mikroabszessen in den Biopsien. Die serologische Eosinophilie korrelierte zeitlich mit der Medikamenteneinnahme. Andere Organe waren nicht beteiligt. Die Symptomatik verbesserte sich nach Absetzen der Medikamente und Verabreichung von Steroiden. Die eosinophile Kolitis ist eine ausgesprochen seltene Erkrankung mit bisher noch unklarem Wirkmechanismus. Bei Patienten mit abdominellen Schmerzen, Diarrhoe und peripherer Eosinophilie unter einer Therapie mit Pregabalin oder Gabapentin sollte der Verdacht auf eine eosinophile Kolitis gestellt werden. 


\section{Introduction}

Eosinophilic colitis (EC) is a rare clinical condition that most frequently affects neonates and young adults and is one of a spectrum of eosinophilic gastrointestinal disorders $[1,2]$. Eosinophilic infiltration of the gastrointestinal tract can be seen in a number of diseases such as parasitic and bacterial infections, inflammatory bowel disease, hypereosinophilic syndrome, Churg-Strauss syndrome, connective tissue disorders, myeloproliferative neoplasms, and drug hypersensitivity [3-5]. Less commonly, eosinophilic infiltration of the gut occurs in the absence of the aforementioned causes, in which case it is considered a primary disorder, the colon being the least frequently involved site in the gastrointestinal tract. Clinical presentation of EC is usually nonspecific and depends on the layer of the intestinal wall that eosinophils have infiltrated. Abdominal pain, non-bloody and bloody diarrhoea, and significant weight loss are the hallmarks of EC. EC may also present acutely and lead to severe and fatal complications such as haemorrhage and perforation [6]. Other symptoms include nausea, vomiting, obstruction, malabsorption, and ascites. Despite the informative role of endoscopy, crosssectional imaging and biochemistry testing, histopathological confirmation of the disease is required usually in the form of a mucosal biopsy.

Drug reaction with eosinophilia and systemic symptoms (DRESS) syndrome is a rare, druginduced reaction characterized by fever, rash, lymphadenopathy, elevated liver enzymes, and leukocytosis with eosinophilia [7,8]. DRESS syndrome demonstrates a long latency period between exposure and disease onset when compared with other drug reactions, and complicates recognition and diagnosis. Among many other medications, gabapentin and pregabalin have been implicated as possible causative agents. The exact pathogenesis of this condition remains unclear, but a drug-specific immune response and herpes virus reactivation are considered key factors [9]. Eosinophilic pneumonitis has been described during therapy with gabapentin [10]. To the best of our knowledge, this is the first case of EC secondary to gabapentin and pregabalin, on a background of chronic fatigue syndrome and functional abdominal pain, described in the medical literature.

\section{Case Report}

A bed-bound 18-year-old girl with a history of incapacitating postural orthostatic tachycardia syndrome, severe chronic fatigue syndrome/myalgic encephalopathy, chronic total body pain, joint hypermobility syndrome, and recurrent urinary tract infections was admitted as an inpatient for enteral feeding and physical rehabilitation. On admission her weight was $35.3 \mathrm{~kg}$ and was fed through a nasojejunal tube. Weight loss was secondary to functional dysphagia, nausea and severe functional dyspepsia postprandial distress syndrome preventing the patient from eating normal size meals. Previous upper gastrointestinal endoscopy had been reassuringly normal. Her medical background included laparascopic appendicectomy, juvenile ostechondritis of her spine, intervertebral disc prolapse and eczema. Chronic fatigue symptoms started soon after her human papilloma virus vaccination. Anorexia nervosa had been previously excluded by a psychiatric consultant. Neurological and metabolic investigations ruled out other causes for her profound fatigue.

The multi-professional approach included regular input from psychology, physiotherapy, occupational therapy, and dietetics. The complex inpatient pain team, adolescent chronic fatigue consultant and neurogastroenterology consultant were involved in her case. Severe musculoskeletal pain and hyperalgesia prevented any form of physical rehabilitation thus treatment with gabapentin was initiated. Gabapentin is an antiepileptic agent and analogue of $\gamma$-amino butyric acid (GABA) with visceral pain reducing activity frequently used in the treatment of neuropathic pain. Within five days, the patient developed severe pain in the right 
iliac fossa followed by explosive, malodorous diarrhoea. Faecal calprotectin was abnormally raised $459 \mu \mathrm{g} / \mathrm{g}(0-60)$. Abdominal symptoms limited enteral feed tolerance due to severe abdominal pain and bloating. A small bowel MRI (Figure 1A) showed severe right sided colitis, clearly abnormal in comparison with MRI images obtained soon after admission. A colonoscopy demonstrated patchy erythema from the sigmoid to the caecum, this being most marked inflammation in the right colon and caecum with multiple mucosal ulcers (Figure 1B and Figure 2). The terminal ileum was oedematous but not erythematous. Colonic biopsies showed a florid eosinophilic inflammation with abscesses raising the possibility of a medication-associated colitis. Mast cells were present on biopsies but were not indicative of a mast cell infiltration disorder. Faecal cultures were negative for ova, cysts, parasites, shigella, campylobacter, salmonella, entamoeba histolytica, giardia, cryptosporidium, C. difficile, norovirus G1 \& G2, rotavirus and faecal adenovirus 40/41. She had negative amoebic, schistosoma and strongyloides serology. A vasculitis screen was negative. Her eosinophil count peaked at $1.93 \times 10^{9} / \mathrm{L}(0.0-0.4)$. Due to the rare reported cases of gabapentin associated eosinophilia and eosinophilic syndromes or drug-induced colitis [3-5,11-15], gabapentin was stopped after one and a half months of treatment. The patient's eosinophil count partially improved but remained high. However, the faecal calprotectin dropped to $31 \mu \mathrm{g} / \mathrm{g}$. No steroids were given at the time. Small bowel ultrasound showed resolution of inflammation weeks after cessation of treatment with gabapentin.

In the context of radiological improvement and improved faecal calprotectin, other agents were tried as treatment for her abdominal pain including opioids. However, the neuropathic agent pregabalin was added to her treatment by a different medical team in order to prevent opioid therapy escalation. Pregabalin again exacerbated her abdominal pain and her diarrhoea recurred. Faecal calprotectin was raised again to $176 \mu \mathrm{g} / \mathrm{g}$ and a repeat colonoscopy showed patchy inflammatory pattern most pronounced within the caecum and proximal transverse colon, with milder changes present within the ascending and mid-transverse colon. The ileocaecal valve was inflamed but the terminal ileum was normal apart from some mild lymphoid hyperplasia. Biopsies confirmed the presence of an EC. Haematology review for possible hypereosinophilic syndrome was felt be low likelihood, in the context of no systemic disease in imaging, an obvious causative agent and hence bone marrow aspirate was deemed not necessary. Throughout her admission, she was on cetirizine $10 \mathrm{mg}$ once daily and chlorphenamine $4 \mathrm{mg}$ four times per day as needed; they did not affect eosinophil count.

Recurrence of the EC compromised again the patient's ability to tolerate enteral feeding and so treatment with steroids was initiated. This lead to a quick symptomatic improvement. Both eosinophilia and abdominal symptoms improved dramatically. The patient was eventually maintained on $55 \mathrm{~mL} / \mathrm{hr}$ for 20 hours nasojejunal feeding reaching a weight of $46.5 \mathrm{~kg}$ (BMI $18.8 \mathrm{~kg} / \mathrm{m}^{2}$ ). Small bowel ultrasound performed two months later confirmed no presence of colitis and repeat faecal calprotectin was $27 \mu \mathrm{g} / \mathrm{g}$.

\section{Imaging, bloods and histology}

The initial small bowel MRI showed an abnormal colon with mural and perimural oedema, wall thickening and restricted diffusion, most notably in the transverse, but also affecting the caecum and ascending. The descending colon, sigmoid and rectum appeared normal (Figure 1A). During pregabalin use, a small bowel ultrasound showed mild thickening involving the caecum, proximal ascending colon and terminal ileum in keeping with recurrent inflammation (Figure 1C). Figure 3 shows the eosinophilia pre- and during neuropathic agents' administration as well as after stopping both agents and starting steroids. Other inflammatory markers were essentially unremarkable. Faecal calprotectin was raised. Blood films were negative for a haematological disorder. Random colonic biopsies showed preserved crypt architecture but there was a patchy infiltrate of eosinophils in the lamina propria (up to 2000 
eosinophils per $\mathrm{mm}^{2}$ ) focally extending into crypt epithelium. No parasites or granulomas were seen. Mast cell counts were up to 30 mast cells per high-power field in inflamed areas (mean 100 eosinophils per $\mathrm{mm}^{2}$ ) on CD117 staining.

Figure 1. A. Small bowel MRI showing inflamed colon during gabapentin use. B. Colonoscopy showing inflammation during gabapentin use. C. Small bowel ultrasound showing inflamed caecum during pregabalin use. D. Colonoscopy showing erythematous colon during pregabalin use.

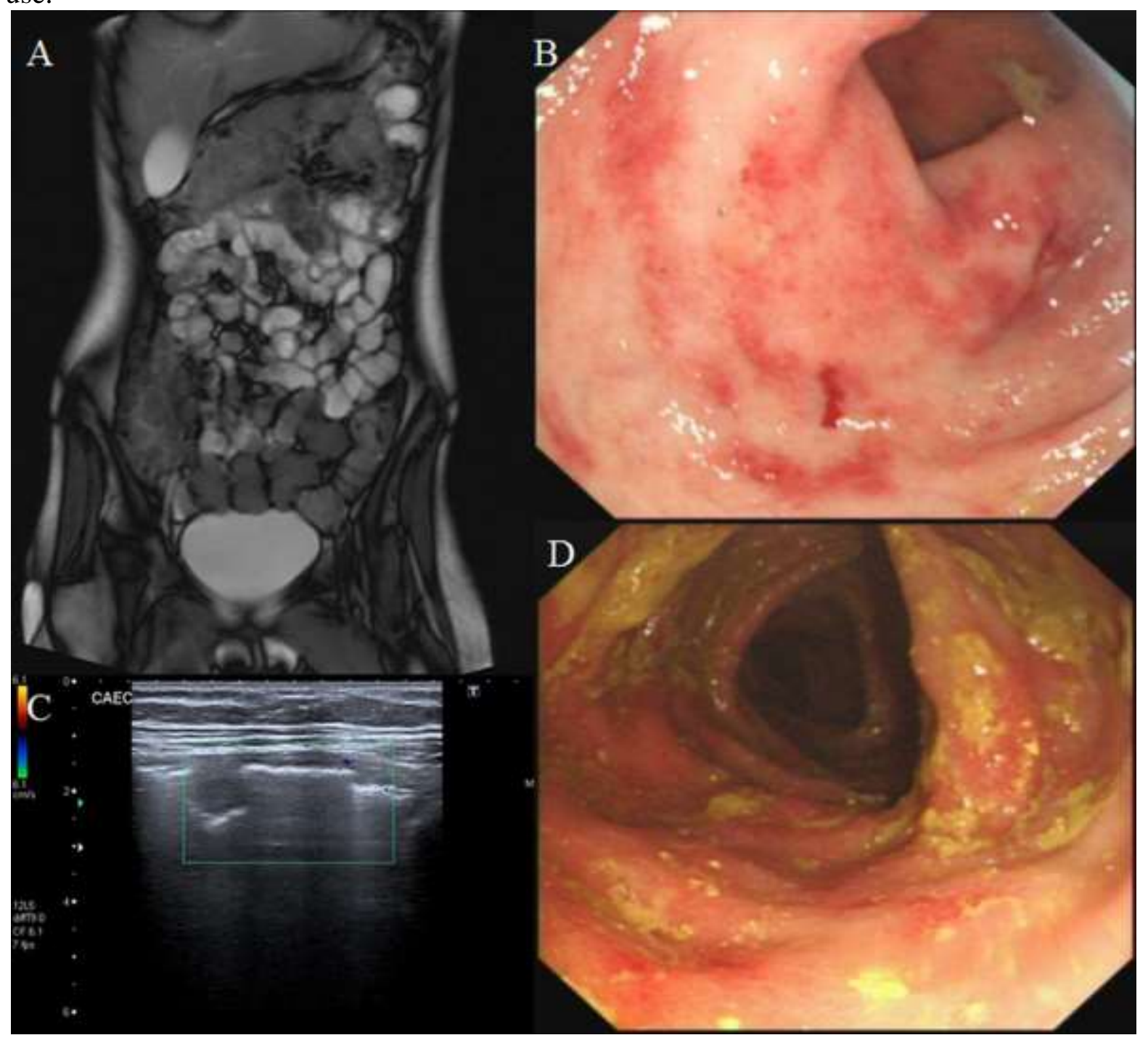


Figure 2. Histology. Random colonic biopsies showed preserved crypt architecture (A) but there was a patchy infiltrate of eosinophils in the lamina propria (up to 2000 eosinophils per $\mathrm{mm} 2$ ) focally extending into crypt epithelium (B). No parasites or granulomas were seen.
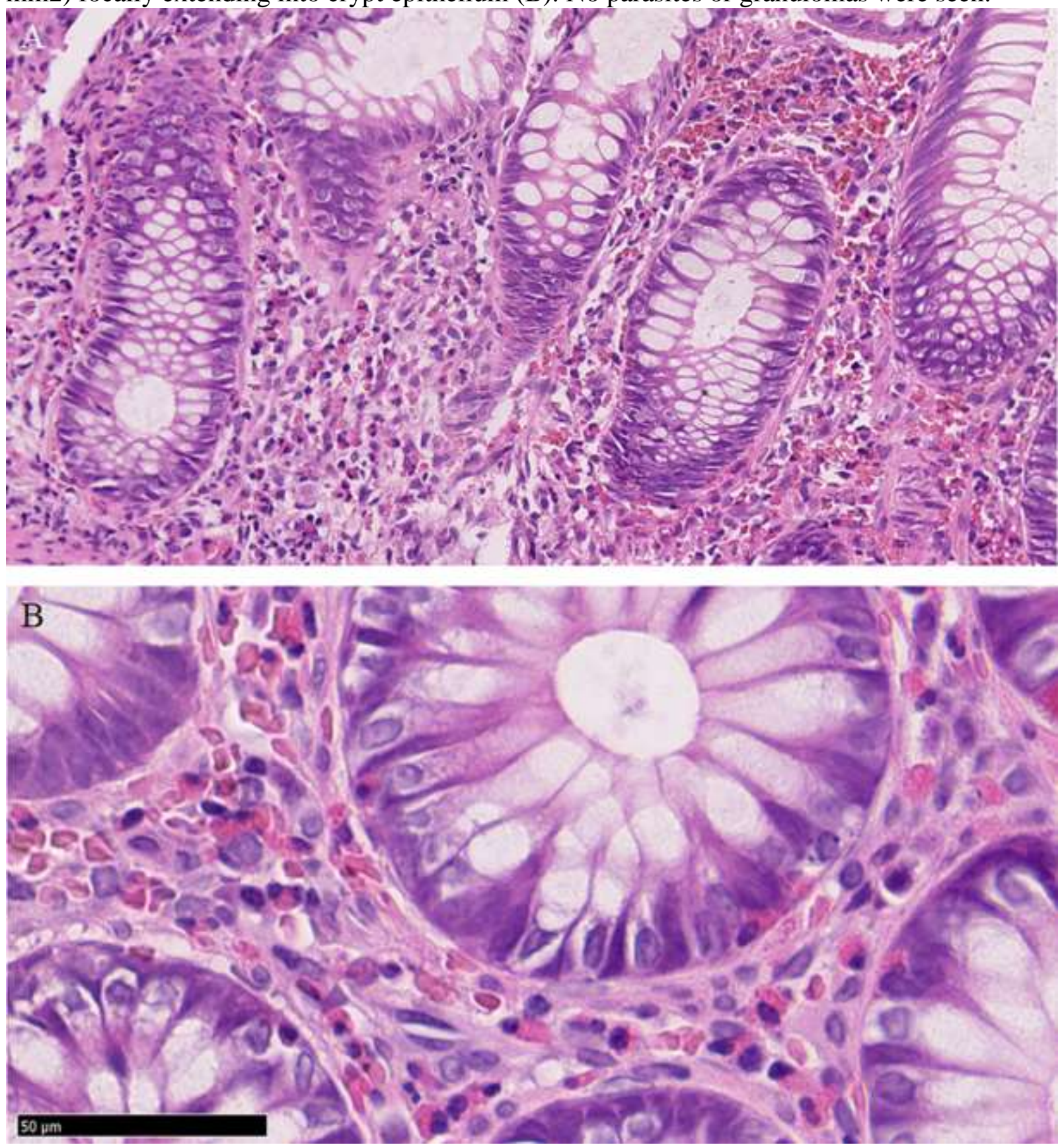
Figure 3. Eosinophil, neutrophil and white cell count.
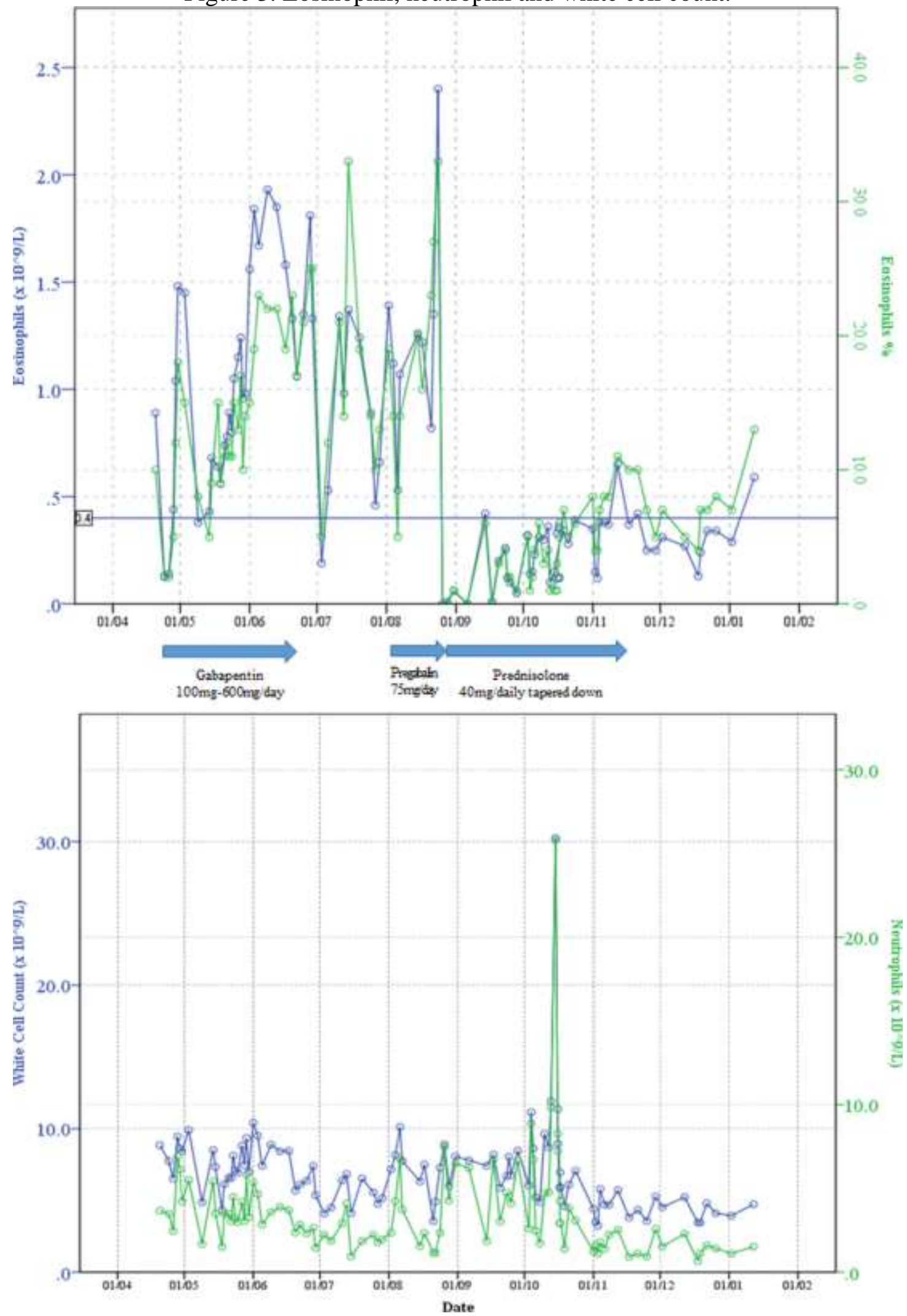


\section{Discussion}

To our knowledge, this is the first case in the literature relating EC to the neuropathic agents gabapentin and pregabalin. Pregabalin and gabapentin share a similar mechanism of action, inhibiting calcium influx and subsequent release of excitatory neurotransmitters. Both pregabalin and gabapentin are antiepileptic medications that bare structural resemblance to GABA, though neither agent has activity in GABA's neuronal systems. They are frequently used as neuropathic agents irrespective of the cause of the neuropathic pain and are frequently prescribed for the management of chronic pain syndromes, including fibromyalgia [16]. Recent studies suggest the combination of pregabalin and duloxetine in the management of pain associated with chronic musculoskeletical pain typical of fibromyalgia [17]. Inhibition of both visceral nociception and gastrointestinal function by gabapentin and pregabalin is supported by evidence form animal studies while in humans, reduction of rectal sensitivity to balloon distension, anti-allodynia, and anti-hyperalgesia were evident in irritable bowel syndrome patients [18].

Acute abdominal pain on a background of chronic abdominal pain in a patient with polypharmacy can prove a serious diagnostic challenge. Eosinophilic gastrointestinal disorders have been described with clozapine, carbamazepine, rifampicin, non-steroidal antiinflammatory drugs, tacrolimus, and gold [2]. The literature describes 75 cases of EC related to various other medications (e.g. chemotherapy, anti-epileptics, antibiotics), food allergies or other inflammatory disease (bowel and non-bowel); no clear mechanism is identified $[4,6,11,13]$. However, EC has never been described in the literature in association with neuropathic agents [3-5]. Gabapentin and pregabalin have both been associated with the hypersensitivity syndrome (fever, rash, tender lymphadenopathy, hepatitis, and eosinophilia) [19-21] and hepatotoxicity [22-24] or cholestasis [25] in rare occasions.

Our case was further complicated by the presence of chronic fatigue syndrome and functional abdominal pain. It is well described in the literature that the immune system may play a role in functional bowel disorders with mast cell activation, eosinophil and intraepithelial lymphocyte infiltrate [26]. Previous studies have shown higher concentrations of eosinophils and mast cells in gastrointestinal mucosa of patients with functional bowel disorders compared to healthy controls [26,27]. However, endoscopic appearances were normal and the severity of the infiltrate seen in our patient could not be simply attributed to irritable bowel syndrome. The case described above had chronic fatigue syndrome, functional abdominal pain, recurrent urinary tract infections and postural orthostatic tachycardia syndrome. However, the sudden change in bowel habit and eosinophilia that had developed during the admission, indicated that medical intervention had possibly caused this new clinical presentation. The factors that determined diagnosis were synchrony in elevation of eosinophils and use of neuropathic agents, atypical histological appearance, and symptom relief and radiological improvement when agents stopped.

EC treatment remains controversial. When aetiological factors are demonstrated, treatment should be directed to address those (e.g. diet, medications). Steroids remain the mainstay therapy in moderate/severe cases with usual treatment being prednisolone 40mg once daily tapered down over a course of weeks or months. Dietary interventions are equally important, particularly in the paediatric population. The uses of elimination and elemental diets have been shown to improve clinical symptoms and reduce mucosal eosinophils in patients with eosinophilic oesophagitis and a similar treatment strategy has also been utilized in the treatment of EC $[3,28]$. Successful treatment with dietary therapy may also be influenced by distribution of eosinophilic infiltration, age of the patient, and ability to comply with dietary restriction [3]. In our case, an exclusive elemental enteral diet in combination with steroids led to a great improvement of symptoms, with weight gain and objective disease remission. 
In conclusion, EC is an exceptionally rare entity and its mechanism of action is still unclear. Suspicion of EC should be raised if a patient presents with abdominal pain, nausea, vomiting, early satiety, diarrhoea, weight loss or ascites associated with peripheral eosinophilia). Different drugs have been proposed to potential lead to EC. This paper demonstrates that, the frequently used agents gabapentin and pregabalin should be added to this list. Taking into consideration their increasing use in functional gut disorders which can present with abdominal pain and diarrhoea, a superimposed eosinophilic colitis might be extremely difficult to identify. Awareness of iatrogenic eosinophilic colitis caused by these agents is important to prevent unnecessary diagnostic delays.

\section{Acknowledgements}

We thank two anonymous reviewers for their comments that helped improve the manuscript and Lara Gamper who helped with the writing of the German abstract.

\section{References}

1. Shifflet A, Forouhar F, Wu GY. Eosinophilic digestive diseases: eosinophilic esophagitis, gastroenteritis, and colitis. Journal of the Formosan Medical Association = Taiwan yi zhi 2009; 108: 834-843

2. Okpara N, Aswad B, Baffy G. Eosinophilic colitis. World journal of gastroenterology 2009; 15: 2975-2979

3. Uppal V, Kreiger P, Kutsch E. Eosinophilic Gastroenteritis and Colitis: a Comprehensive Review. Clinical reviews in allergy \& immunology 2016; 50: 175-188

4. Dionisio de Sousa IJ, Bonito N, Pais A et al. Eosinophilic colitis. BMJ case reports 2016; 2016:

5. Yozgat A, Colak A, Koseoglu HT et al. Eosinophilic colitis under etanercept. Acta gastro-enterologica Belgica 2015; 78: 439-440

6. Alfadda AA, Storr MA, Shaffer EA. Eosinophilic colitis: epidemiology, clinical features, and current management. Therapeutic advances in gastroenterology 2011; 4: 301-309

7. Spriet S, Banks TA. Drug reaction with eosinophilia and systemic symptoms syndrome. Allergy Asthma Proc 2015; 36: 501-505

8. Shiohara T, Kano Y. Drug reaction with eosinophilia and systemic symptoms (DRESS): incidence, pathogenesis and management. Expert Opin Drug Saf 2017; 16: 139-147

9. Roujeau JC, Dupin N. Virus Reactivation in Drug Reaction with Eosinophilia and Systemic Symptoms (Dress) Results from a Strong Drug-Specific Immune Response. J Allergy Clin Immunol Pract 2017; 5: 811-812

10. Eshki M, Allanore L, Musette P et al. Twelve-year analysis of severe cases of drug reaction with eosinophilia and systemic symptoms: a cause of unpredictable multiorgan failure. Arch Dermatol 2009; 145: 67-72

11. Jensen ET, Martin CF, Kappelman MD et al. Prevalence of Eosinophilic Gastritis, Gastroenteritis, and Colitis: Estimates From a National Administrative Database. Journal of pediatric gastroenterology and nutrition 2016; 62: 36-42

12. Franco AI, Escobar L, Garcia XA et al. Mesalazine-induced eosinophilic pneumonia in a patient with ulcerative colitis disease: a case report and literature review. International journal of colorectal disease 2016; 31: 927-929 
13. Kocak G, Kocak E, Akbal E et al. Eosinophilic colitis: a rare cause of lower gastrointestinal bleeding in an elderly adult. Journal of the American Geriatrics Society 2014; 62: 2016-2017

14. Farooqi A. Eosinophilic colitis mimicking caecal malignancy. Journal of Ayub Medical College, Abbottabad : JAMC 2014; 26: 408-409

15. Alfadda AA, Shaffer EA, Urbanski SJ et al. Eosinophilic colitis is a sporadic selflimited disease of middle-aged people: a population-based study. Colorectal disease : the official journal of the Association of Coloproctology of Great Britain and Ireland 2014; 16: 123-129

16. Smith MT, Moore BJ. Pregabalin for the treatment of fibromyalgia. Expert Opin Pharmacother 2012; 13: 1527-1533

17. Arnold LM, Clauw DJ, Dunegan LJ et al. A framework for fibromyalgia management for primary care providers. Mayo Clin Proc 2012; 87: 488-496

18. Gale JD, Houghton LA. Alpha 2 Delta (alpha(2)delta) Ligands, Gabapentin and Pregabalin: What is the Evidence for Potential Use of These Ligands in Irritable Bowel Syndrome. Front Pharmacol 2011; 2: 28

19. Ragucci MV, Cohen JM. Gabapentin-induced hypersensitivity syndrome. Clin Neuropharmacol 2001; 24: 103-105

20. Goldman J, Duval-Modeste AB, Musette P et al. [Drug-induced hypersensitivity syndrome due to gabapentin]. Ann Dermatol Venereol 2008; 135: 230-232

21. Bamanikar A, Dhobale S, Lokwani S. Pregabalin hypersensitivity in a patient treated for postherpetic neuralgia. Indian J Pharmacol 2013; 45: 522-523

22. Lasso-de-la-Vega MC, Zapater P, Such J et al. Gabapentin-associated hepatotoxicity. The American journal of gastroenterology 2001; 96: 3460-3462

23. Dogan S, Ozberk S, Yurci A. Pregabalin-induced hepatotoxicity. European journal of gastroenterology \& hepatology 2011; 23: 628

24. Sendra JM, Junyent TT, Pellicer MJ. Pregabalin-induced hepatotoxicity. Ann Pharmacother 2011; 45: e32

25. Richardson CE, Williams DW, Kingham JG. Gabapentin induced cholestasis. BMJ 2002; 325: 635

26. Wouters MM, Vicario M, Santos J. The role of mast cells in functional GI disorders. Gut 2016; 65: 155-168

27. Kim SE, Chang L. Overlap between functional GI disorders and other functional syndromes: what are the underlying mechanisms? Neurogastroenterol Motil 2012; 24: 895-913

28. Spergel JM, Rothenberg ME, Collins MH et al. Reslizumab in children and adolescents with eosinophilic esophagitis: results of a double-blind, randomized, placebocontrolled trial. J Allergy Clin Immunol 2012; 129: 456-463, 463 e451-453 